\title{
Presence of Multifractality in High-Energy Nuclear Collisions
}

\author{
M. I. Haque1, M. Tariq2 ${ }^{2}$ Tahir Hussain ${ }^{3}$ \\ ${ }^{1}$ Department of Kulliyat, AK Tibbiya College, Aligarh Muslim University, Aligarh, India \\ ${ }^{2}$ Department of Physics, Aligarh Muslim University, Aligarh, India \\ ${ }^{3}$ Department of Applied Physics, Aligarh Muslim University, Aligarh, India \\ Email: mihaqueamu@gmail.com, mtariqamu@gmail.com, tahir.phys@gmail.com
}

Received 1 September 2014; revised 25 September 2014; accepted 13 October 2014

Copyright (C) 2014 by authors and Scientific Research Publishing Inc.

This work is licensed under the Creative Commons Attribution International License (CC BY).

http://creativecommons.org/licenses/by/4.0/

cC) (7) Open Access

\begin{abstract}
In the present study an attempt is made to examine multifractality in multiparticle production in relativistic nuclear collisions; multifractality is investigated in $14.5 \mathrm{~A} \mathrm{GeV} / \mathrm{c}^{28} \mathrm{Si}$-nucleus collisions. For this, $G_{q}$-moments are calculated and variations of $\ln \left\langle G_{q}\right\rangle$ with $-\ln \delta \eta$ are looked into. Values of mass exponents, $t_{q}$, and generalised dimensions, $D_{q}$, are obtained. Analysis of multifractal moments reveals that multiplicity fluctuations are of dynamical nature.
\end{abstract}

\section{Keywords}

\section{Multifractal Moments, Dynamical Fluctuations, Quark-Gluon Plasma, Emulsion}

\section{Introduction}

Analysis of high-energy heavy-ion collisions [1]-[4] offers a unique opportunity to investigate occurrence of dynamical fluctuations [5]-[7] in A-A collisions. To understand the real dynamics of multiparticle production, multifractality is envisaged to become an important tool for both theoretician and experimentalist. Intermittency and multifractality in turbulent fluids have been extensively studied [8]. It was suggested that multifractal analysis is carried out by calculating multifractal moments, $G_{q}$, of the multiplicity distributions in a given pseudorapidity $(\eta)$ space. The main purpose of adopting multifractal moments, $G_{q}$, approach is to explain multifractality and self-similarity in multiparticle production in relativistic nucleus-nucleus collisions. However, $G_{q}$ moments are greatly influenced by statistical fluctuations in the case of events having lower multiplicities. It is worth mentioning that if the particle production process exhibits self-similar behavior, a modified form of $G_{q}{ }^{-}$ moment is used by introducing a step function [2], which leads to power-law dependence on the phase space bin size. Importance of multifractal analysis of high-energy nuclear collision data lies in the fact that these moments 
can be calculated for the negative values of the order of moments, $q$, also, whereas factorial moments are defined only for positive integral values of the order of moments. High-energy heavy-ion collisions are considered to be an ideal site for creating the conditions for producing Quark-Gluon Plasma (QGP) and dynamical fluctuations are one of the most reliable signals of QGP formation. Fluctuations in multiplicity and pseudorapidity distributions [9] are the most significant approaches to study nuclear matter produced in these collisions. Using the calculated values of various moments, non-statistical fluctuations in high-energy nuclear interactions can be investigated. It may be noted that multifractality may play an important role for searching the existence of dynamical fluctuations in the multipaticle production.

\section{Mathematical Formalism}

In order to study multifractality, a selected pseudorapidity interval, $\Delta \eta$ is partitioned into $M$ bins of equal size $\delta \eta=\Delta \eta / M$. Let $n_{j}$ be the number of particles lying in $j^{\text {th }}$ bin, then multifractal moments, $G_{q}$, may be calculated [1] [10] using:

$$
G_{q}=\sum_{j}\left(p_{j}\right)^{q}
$$

where quantity $p_{j}$ is defined as $p_{j}=n_{j} / n$ and $n$ be the total number of particles.

In the above expression the summation is carried over non-empty bins only. For a given data sample, averaging is done over all the events comprising the total number of events, $N_{\text {evt }}$, the average value of multifractal moments, $\left\langle G_{q}\right\rangle$ is calculated from:

$$
\left\langle G_{q}\right\rangle=\frac{1}{N_{\text {evt }}} \sum_{1}^{N_{\text {evt }}} G_{q}
$$

If rapidity distribution possesses fractal nature, a power-law behavior of $\left\langle G_{q}\right\rangle$ of the following type should be observed over a small pseudorapidity range, $\delta \eta$ :

$$
\left\langle G_{q}\right\rangle \propto(\delta \eta)^{t_{q}}
$$

where $t_{q}$ are known as mass exponents.

The resulting linear dependence of $\ln \left\langle G_{q}\right\rangle$ on $-\ln \delta \eta$ may be used to determine the values of $t_{q}$ making use of the following relationship:

$$
t_{q}=\lim _{\delta \eta \rightarrow 0} \frac{\Delta \ln \left\langle G_{q}\right\rangle}{\Delta \ln \delta \eta}
$$

The generalized dimensions, $D_{q}$, are considered to contain useful property regarding fractals occurring in multiparticle production in relativistic nuclear collisions. Generalized dimensions are defined as:

$$
D_{q}=\frac{t_{q}}{q-1}
$$

Increase in the value of $D_{q}$ with $q$ is said to describe the pattern as multi fractal, whereas constancy of $D_{q}$ would point towards monofractality.

\section{Experimental Details}

We have analyzed data set comprising 555 events produced in $14.5 \mathrm{~A} \mathrm{GeV/c}{ }^{28}$ Si-nucleus collisions. Data sample include collisions with $n_{h} \geq 0$, where $n_{h}$ represent the number of charged particles produced with relative velocities, $\beta \leq 0.7$. Experimental results have been compared with the corresponding results for the data generated using Lund model, FRITIOF and Monte Carlo simulation.

\section{Results and Discussion}

\subsection{Study of $\ln \left\langle G_{q}\right\rangle$ as a Function of $-\ln (\delta \eta)$}

Figure 1 shows the variations of $\ln \left\langle G_{q}\right\rangle$ with $-\ln \delta \eta$ for the experimental data on $14.5 \mathrm{~A} \mathrm{GeV/c}{ }^{28} \mathrm{Si}$-nuc- 
leus collisions; these variations are studied for three groups of targets namely, CNO, emulsion and AgBr groups of nuclei for various classes of interactions. It may be emphasized that multifractal moments show linearly increasing trend with decreasing bin width, $\delta \eta$, for all the three groups of the targets; this linearly increasing behavior which is shown over a large interval of $-\ln \delta \eta$ for positive order of the moments, $q$, in comparison to the ones for negative $q$ values. For negative values saturating trend is discernible with decreasing $\delta \eta$. The only reason for this behavior appears to be the fact that particle multiplicity will decrease with decreasing bin width, $\delta \eta$ [11]. Multifractal moments for CNO group of targets saturates a little earlier in comparison to those for AgBr nuclei. This linearly increasing nature demonstrated by multifractal moments in the rapidity space indicates the presence of self-similarity. To investigate the dynamical fluctuations using multifractal moments, experimental results are compared with the Monte Carlo generated data sets for $14.5 \mathrm{~A} \mathrm{GeV/c}{ }^{28} \mathrm{Si}$-nucleus collisions. Figure 2 compares the behaviors of $\ln \left\langle G_{q}\right\rangle$ vs $-\ln \delta \eta$ plots for the experimental, FRITIOF and Monte Carlo generated data sets. The variation for the MC simulated data is very smooth as compared to those for the experimental and FRITIOF data samples.
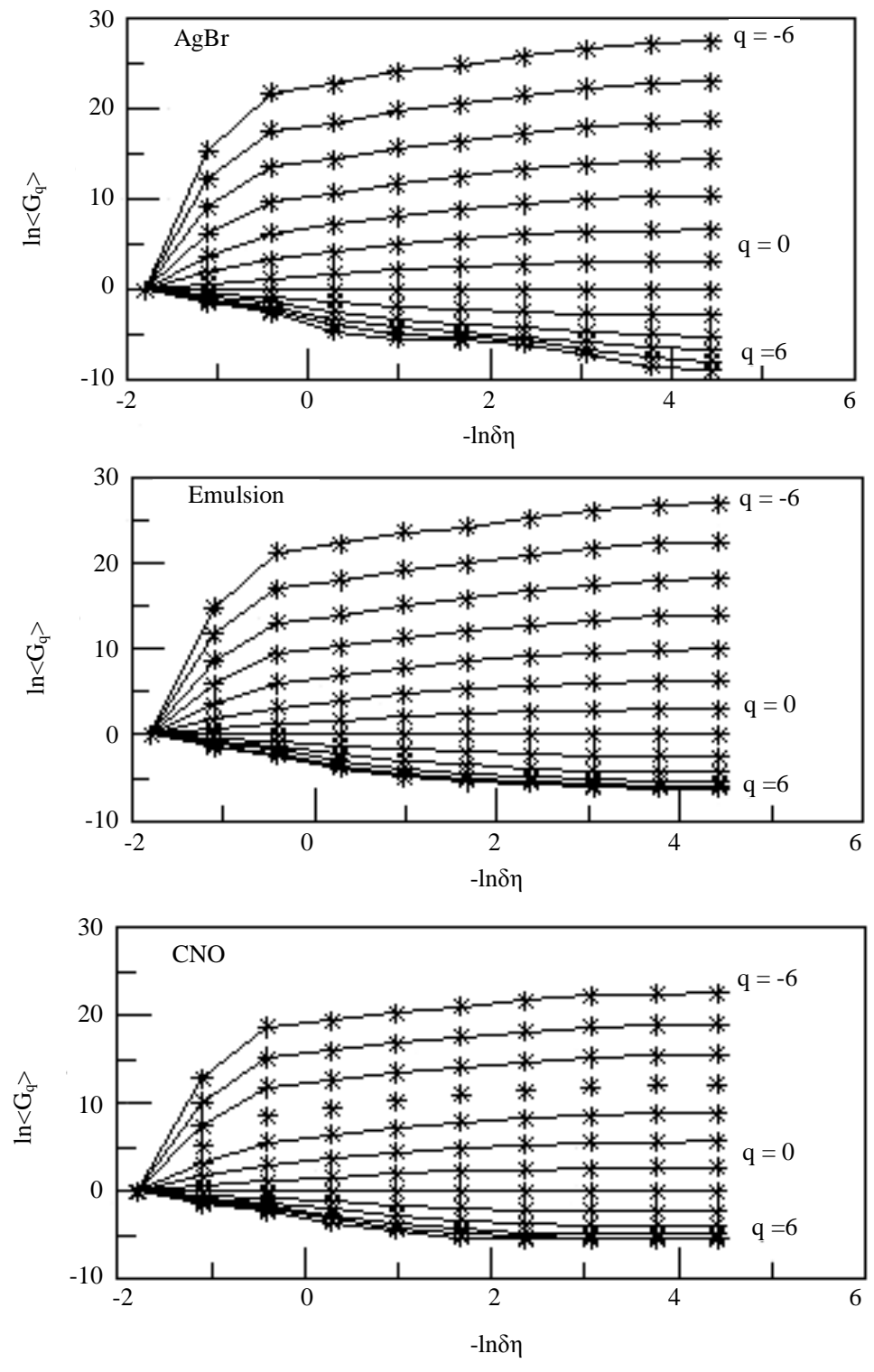

Figure 1. Variations of $\ln \left\langle G_{q}\right\rangle$ with $-\ln \delta \eta$ for the experimental data on 14.5 A GeV/c ${ }^{28}$ Si-nucleus collisions. 

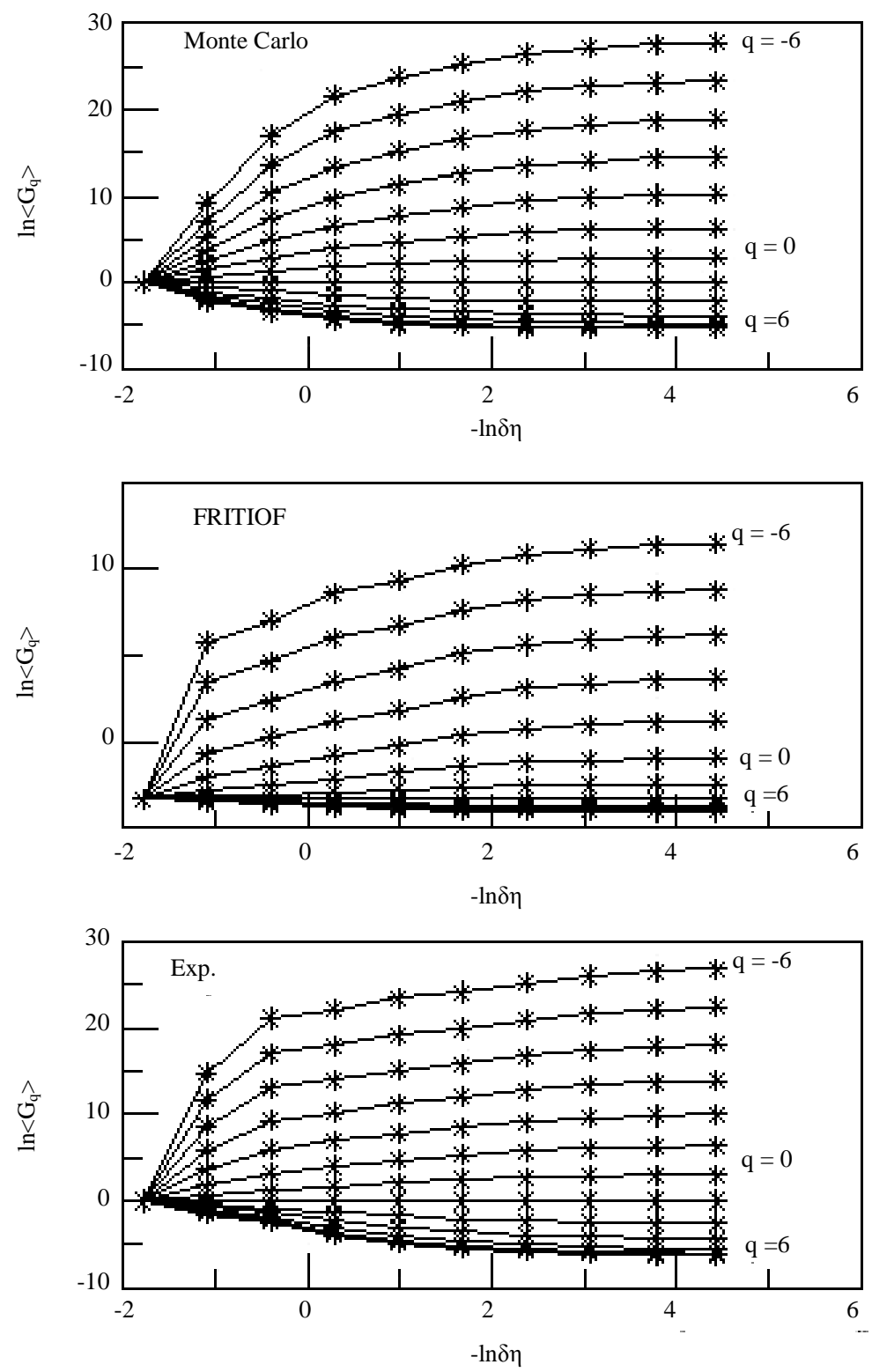

Figure 2. Variations of $\ln \left\langle G_{q}\right\rangle$ with $-\ln \delta \eta$ for the experimental and simulated data samples.

\subsection{Mass Exponents}

Values of the mass exponents, $t_{q}$, are determined by fitting the $\ln \left\langle G_{q}\right\rangle$ versus $-\ln \delta \eta$ plots in the region which exhibits linear behavior. The slopes of these fits give the values of the mass exponents. Figure 3 show the variations of $t_{q}, t_{q}^{\text {stat }}$ and $t_{q}^{\mathrm{dyn}}$ with the order of the moments, where $t_{q}^{\text {stat }}$ represent the slopes for the Monte Carlo generated data and $t_{q}^{\mathrm{dy} q}$ are the dynamical component of the fluctuations; $t_{q}^{\mathrm{dyn}}, t_{q}^{\mathrm{stat}}, t_{q}$ and $q$ satisfy the following relationship:

$$
t_{q}^{\mathrm{dyn}}=t_{q}-t_{q}^{\mathrm{stat}}+q-1
$$

Figure 3 shows that the values of $t_{q}$ increases with increasing $q$. The increasing trends for positive and negative values of $q$ are quite dissimilar. These values depends on $q>0$ and $q<0 . t_{q}^{\mathrm{dyn}}$ are observed to be quite different from $t_{q}$. Figure 4 compares the values of the mass exponents, $t_{q}$, for the experimental and 


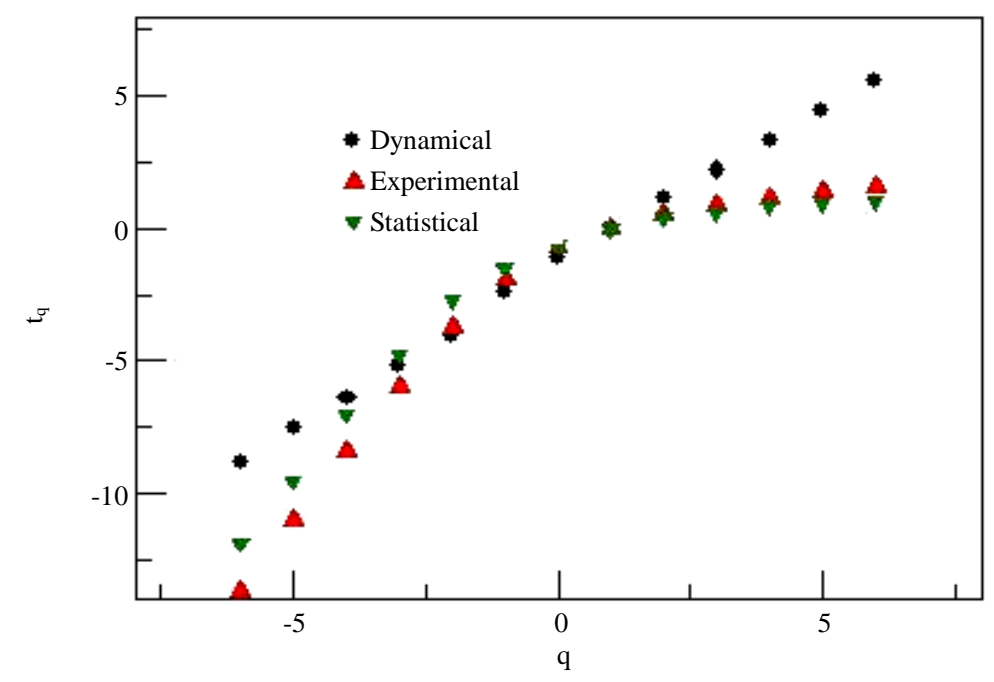

Figure 3. Variations of $t_{q}$, $t_{q}^{\text {stat }}$ and $t_{q}^{\mathrm{dyn}}$ with $q$.

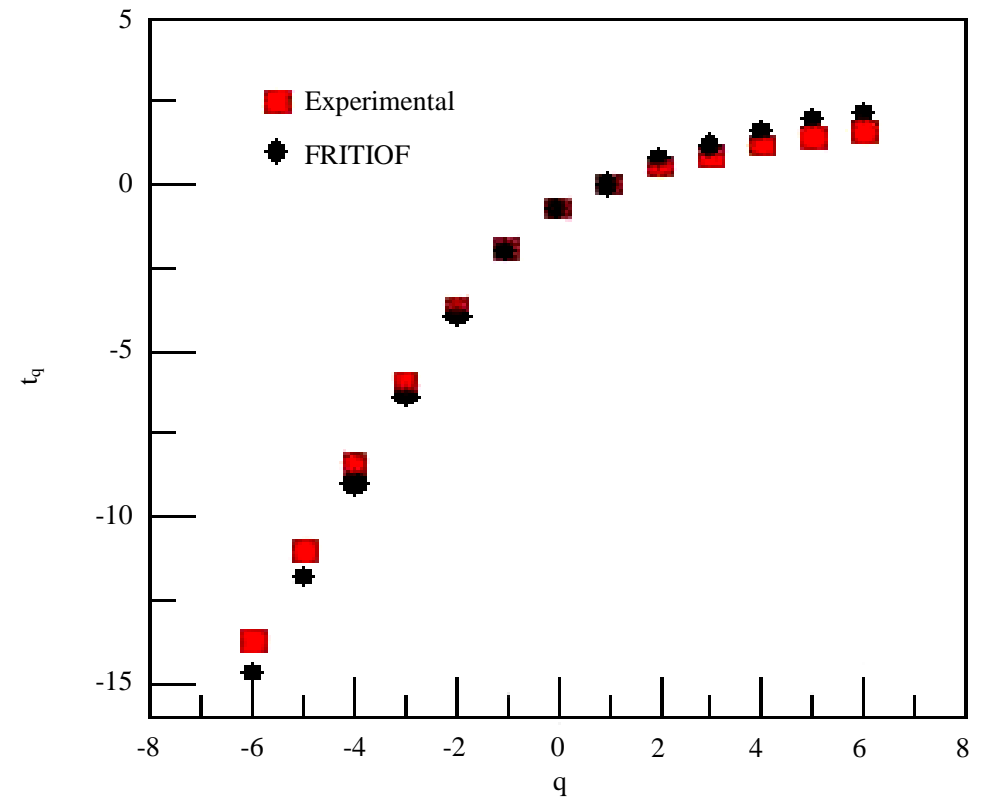

Figure 4. Variations of $t_{q}$ with $q$ for the experimental and FRITIOF data.

FRITIOF generated data. It is noticed that the variations are similar for both the data sets. Again target dependence of the mass exponents, $t_{q}$, which are shown in Figure 5, indicates that the values of mass exponents $t_{q}$, for $q<1$, have lower values for the heavier targets as compared to those for the lighter ones, for which $q>1$.

\subsection{Generalized Dimensions}

Figure 6 exhibits the variations of the generalized dimensions, $D_{q}$, with $q$ for the three categories of targets. From the figure it is clear that the values of $D_{q}$ for higher values of $q$ are relatively lower in comparison to those for the lower values of $q$. For different orders of the moments, $q$, the values of generalized dimensions are positive and decrease with increasing $q$. This behavior supports the predictions of the multifractal cascade model. It is clear from the Figure 6 that the generalized dimensions have higher values for heavier targets. The high values of generalized dimensions for heavier targets may be attributed to the fact that multiplicity for heavier targets is higher. 


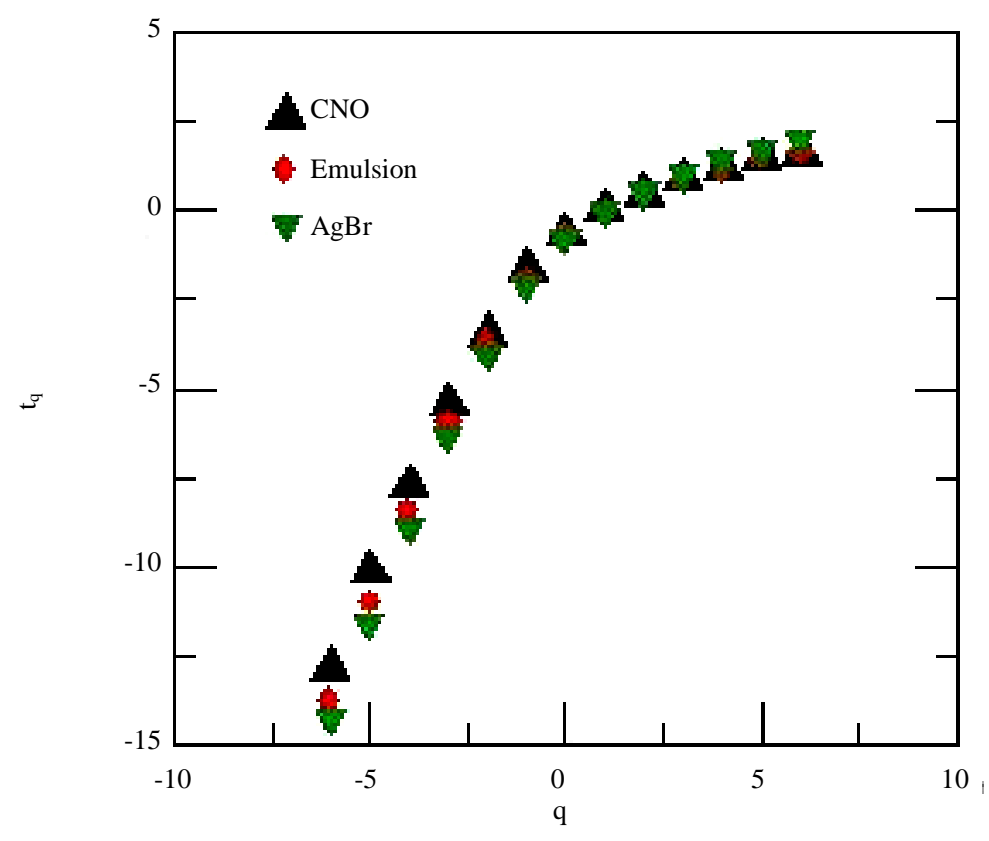

Figure 5. Variations of mass exponents, $t_{q}$, with $q$ for the experimental data on $14.5 \mathrm{~A} \mathrm{GeV/c}{ }^{28} \mathrm{Si}$-nucleus interactions.

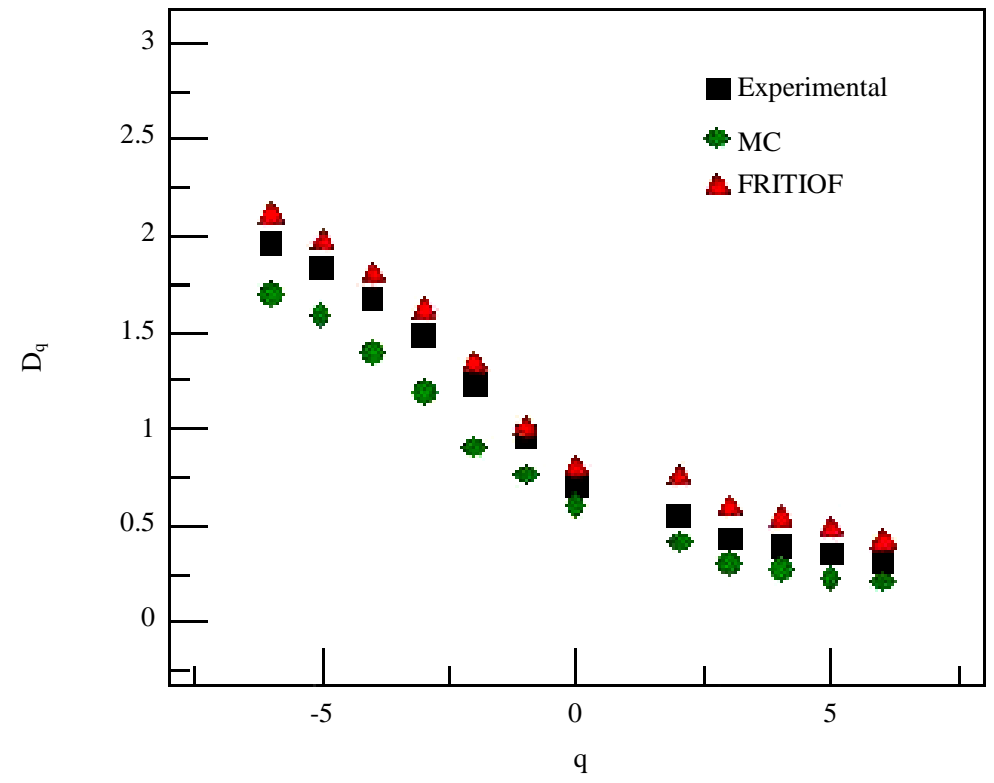

Figure 6. Variations of generalized dimensions, $D_{q}$, with $q$ for the experimental, FRITIOF and Monte Carlo generated data.

\section{Conclusion}

The results of the present study are quite important for drawing meaningful conclusions regarding occurrence of multifractality in multiparticle production in high-energy nucleus-nucleus collisions. It is clearly observed that $\ln \left\langle G_{q}\right\rangle$ first increases linearly with $-\ln \delta \eta$ and then saturates as $\eta$ resolution increases. The nature of variation clearly hints towards power-law behavior of multifractal moments as a function of $\delta \eta$, thereby indicating the presence of multifractality. The variations of mass exponents $t_{q}$, and generalized dimensions $D_{q}$, with $q$ also support the presence of multifractality in the collisions considered in the present study. 


\section{References}

[1] Hwa, R.C. (1990) Physical Review D, 41, 1456. http://dx.doi.org/10.1103/PhysRevD.41.1456

[2] Hwa, R.C. and Pan, J.C. (1992) Physical Review D, 45, 1476. http://dx.doi.org/10.1103/PhysRevD.45.1476

[3] Jain, P.L., Sengupta, K. and Singh, G. (1990) Physics Letters B, 24, 273.

Derado, T., et al. (1992) Physics Letters B, 283, 151.

Albajar, C., et al. (1992) Zeitschrift für Physik C, 56, 37.

[4] Ghosh, D., et al. (1991) Physics Letters B, 272, 5.

Ghosh, D., et al. (1996) Zeitschrift für Physik C, 71, 243.

[5] Schmidt, H.R. and Schukraft, J. (1993) Journal of Physics G: Nuclear and Particle Physics, $19,1705$. http://dx.doi.org/10.1088/0954-3899/19/11/006

[6] Kajantie, K. and Mclerran, L. (1982) Physics Letters B, 119, 203. http://dx.doi.org/10.1016/0370-2693(82)90277-5

[7] Bjorken, J.D. (1983) Physical Review D, 27, 140. http://dx.doi.org/10.1103/PhysRevD.27.140

[8] Mandelbrot, B.B. (1982) The Fractal Geometry of Nature. Freeman, New York.

[9] Muller, B. (1995) Reports on Progress in Physics, 58, 611. Singh, C.P. (1993) Physics Reports, 236, 147.

[10] Chiu, C.B. and Hwa, R.C. (1991) Physical Review D, 43, 100. http://dx.doi.org/10.1103/PhysRevD.43.100

[11] Jain, P.L., Singh, G. and Mukhopadhyay, A. (1992) Physical Review C, 46, 721. http://dx.doi.org/10.1103/PhysRevC.46.721 
Scientific Research Publishing (SCIRP) is one of the largest Open Access journal publishers. It is currently publishing more than 200 open access, online, peer-reviewed journals covering a wide range of academic disciplines. SCIRP serves the worldwide academic communities and contributes to the progress and application of science with its publication.

Other selected journals from SCIRP are listed as below. Submit your manuscript to us via either submit@scirp.org or Online Submission Portal.
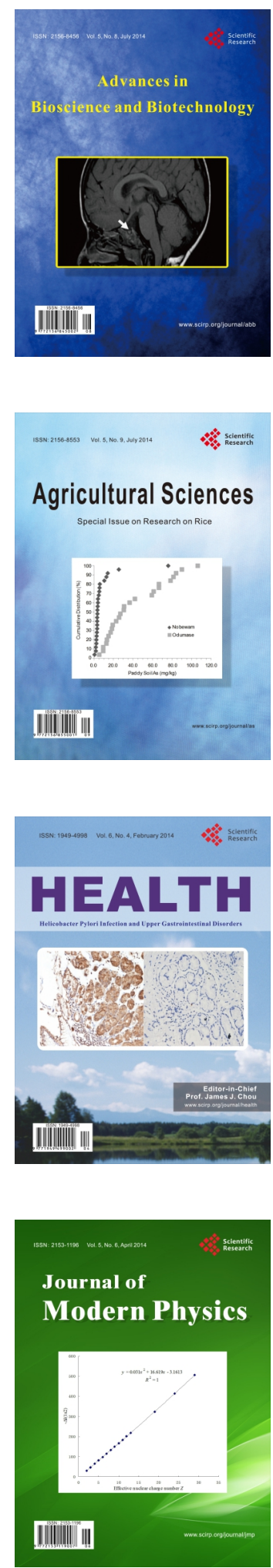
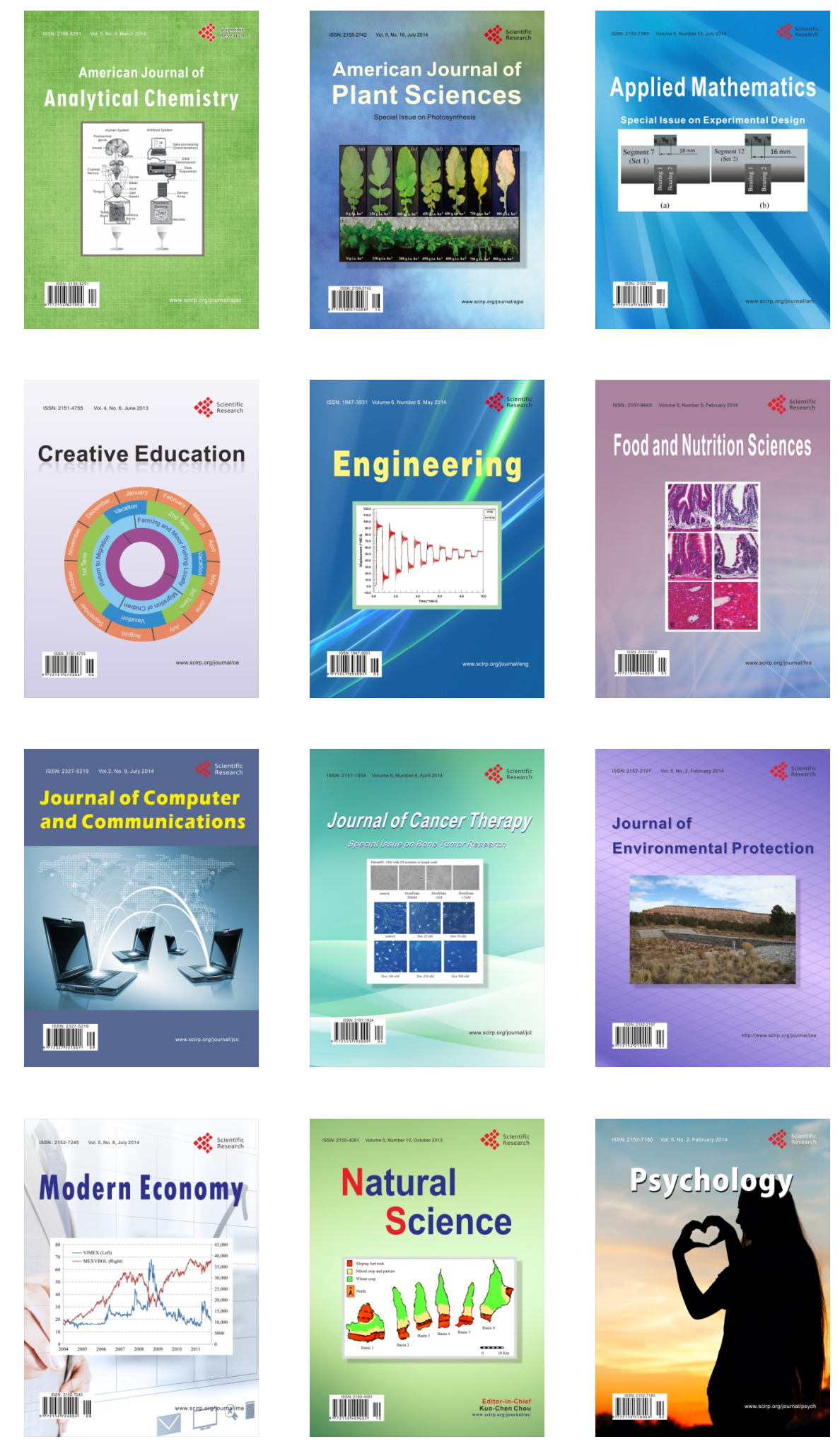